\title{
EFFECT OF SOWING DATES, BIO, ORGANIC AND CHEMICAL FERTILIZATION TREATMENTS ON GROWTH AND PRODUCTION OF INDIAN FENNEL UNDER NORTH SINAI CONDITIONS
}

(Received: 2.11.2011)

\author{
By \\ E. M. Abou El-Ghait, A. O. Gomaa, A. S. M.Youssef, E. M. Atiea* and W. H. Abd-Allah* \\ Horticulture Department, Faculty of Agriculture, Benha University \\ * Medicinal \& Aromatic Plants Department, Desert Research Center, Cairo, Egypt
}

\begin{abstract}
The present study was carried out at El-Sheikh Zowaid Research Station, Desert Research Center, North Sinai Governorate, during the two successive seasons of 2008/2009 and 2009/2010. The objective of this work was to study the effect of sowing date, applying of chemical, bio and organic fertilizers on the vegetative growth and essential oil productivity of Foeniculum vulgare var. panmorium Mill .( Indian fennel type). The highest values of vegetative growth measurements were obtained with treatment F5 (100\% recommended dose+compost $(\mathrm{CM})+$ biofertilizer) which produced the tallest plants, the heaviest fresh and dry weights of herb/plant as compared to other fertilization treatments. The early sowing date ( $7^{\text {th }}$ October) proved to be the most effective one for producing the best vegetative growth in comparison with the other sowing dates. In addition, the highest number of umbels /plant, fruit yield/ plant (g) and per feddan (ton) and weight of 1000 fruits (g) were recorded by using the treatment F5 (100\% recommended dose+compost $(\mathrm{CM})+$ biofertilizer). The highest oil percentage was due to fertilization treatment F4 (biofertilizer +5 ton compost/fed). Whereas, the highest oil yield/plant was obtained from F5 treatment $(100 \%$ recommended dose $)+$ compost $(\mathrm{CM})+$ biofertilizer. Delaying planting date from $7^{\text {th }}$ Oct to $7^{\text {th }}$ Nov decreased both oil percentage and oil yield/plant. The highest values belonged to the early sowing date of $7^{\text {th }}$ October. Moreover, the highest leaf N, P and P contents were gained with the treatment of F5 (100\%recommended dose) + compost (CM) + biofertilizer in both seasons. The main detected component was trans-anethole which is considered an important constituent, followed descendingly by fenchone then estragole. There are other major components including limonene and , $\alpha$-pinene that were found in the tested oil. Delaying sowing date decreased anethol content in the volatile oil. Fenchone percentage in the fennel volatile oil was generally increased with delaying sowing date, and also methyl-chavicol (estragole) was higher in delayed dates, while limonene and $\rho$-cymene recorded the least value with the late sowing date of $7^{\text {th }}$ November.

A dose of $(400 \mathrm{~kg}$ per fed) ammonium sulphate $(20.5 \% \mathrm{~N})+$ super phosphorus, at the rate of $300 \mathrm{~kg}$ calcium super-phosphate $\left(16 \% \mathrm{P}_{2} \mathrm{O}_{5}\right)$ per feddan +Potassium sulfate, at the rate of $100 \mathrm{~kg}$ potassium sulfate $\left(48 \% \mathrm{~K}_{2} \mathrm{O}\right)$ per feddan was recommnded.
\end{abstract}

Key words: anethol, estragole, fertilization, foeniculum, Indian fennel, oil constituents, sowing date.

\section{INTRODUCTION}

Foeniculum vulgare, Mill. is known as fennel, belongs to family Apiaceae or Umbelliferae. Fennel is a strong aromatic perennial shrublet up to $200 \mathrm{~cm}$ high, with feathery leaves and golden yellow flowers. It is native to north Africa, Mediterranean region, southern Europe and Asia. There are two types of oil that are commercially available: bitter fennel oil which is obtained from wild or cultivated Foeniculum vulgare, Mill. var. vulgare, and sweet fennel oil from cultivated Foeniculum vulgare, Mill. var. dulce. Volatile oil is obtained by steam distillation of the crushed ripe fruits. Formacek and Kubeczka (1982), Boulos (1983),Simon (1990), Lawless (1992) and Bown (1995). reported that the essential oil from fennel seed and plant has flavoring, cosmetic, and pharmaceutical uses as stomachic, tonic, carminative, antispasmodic, diuretic, with the approval of the German Office of Health.

It was mentioned that the main constituents of the essential oil of the most important fennel variety (var. dulce) contains anethole (50 to $80 \%$ ), limonene $(5 \%)$, fenchone (5\%), estragole (methyl- 
chavicol), safrole, $\alpha$-pinene $(0.5 \%)$, camphene, $\beta$ pinene, $\beta$-myrcene and p-cymene. In contrast, the uncultivated form (var. vulgare) contains often more essential oil, but since it is characterized by the bitter fenchone (12 to $22 \%$ ), it is of little value and its origin is Mediterranean (Leung 1980), Abou Zeid, E.N. (1988), Lawless (1992), Bown (1995) and Chevallier (1996). Egypt cultivates about 11000 feddans of fennel (Foeniculum vulgare var. vulgare), mostly in Assiut and Qena Governorates. Egyptian type is inferior to the Indian type (Foeniculum vulgare var. panmorium) in the anethol content which is about $20-35 \%$ in the Egyptian variety compared to $60-80 \%$ in the Indian types. Further, Egyptian types have another major drawback which is the high content of estragole (methyl chavicol) which reaches $40-50 \%$ compared to about 14.7-22.6\% in the Indian types.Abd El-Wahab and Mehasen (2009) on Indian fennel plants, found that delaying sowing date decreased anethole content, while fenchone percentage in the fennel volatile oil increased with delaying sowing date. Methyl-chavicol (estragole) was higher in delayed dates, while limonene and $\rho$-cymene recorded the least value with late sowing date under upper Egypt conditions. Estragol is among the substances banned for use in infant formulas and drugs. Some countries such as Germany ban the use of Egyptian fennel in drug manufacturing at large. Accordingly, it is empirical for Egypt to introduce a new variety of fennel with lower estaragol content. Market News Service (MNS) Medicinal Plants \& Extracts (2006) and (ESHEDA 2007).

\section{MATERIALS AND METHODS}

The present study was carried out at El- Sheikh Zowaid Research Station, Desert Research Center, North Sinai Governorate during the two successive seasons of 2008/2009 and 2009/2010. The objective of this work was to study the effect of sowing date, applying of chemical, bio and organic fertilizers on the vegetative growth and essential oil productivity of Foeniculum vulgare var. panmorium (Indian fennel type).

\subsection{Plant material and procedure}

Seeds of Indian fennel were imported from India by (AERI) Institutional Linkage Project the holder of the U.S. Agency for International Development (USAID)-funded AERI/ILA Cooperative.The Egyptian fennel seeds were obtained from the Egyptian Spices and Herb Export Development Association (ESHEDA).

\subsection{Planting dates}

Seeds of Indian fennel were sown on $7^{\text {th }}$ Oct., $21^{\text {st }}$ Oct. and $7^{\text {th }}$ Nov. 2008 and 2009 (in the first and second seasons, respectively). Seeds were sown directly in reclaimed sandy soil in the two seasons. The distance between rows was $75 \mathrm{~cm}$ and $50 \mathrm{~cm}$ between hills. The plants were thinned after germination; two plants per hill (22400 plants/fed ). Drip irrigation was used with drippers (4 liter / hour /hill) both seasons for only one hour every two days.

The analyses of soil and water are shown in Tables 1 and 2. The chemical properties of soil in ppm (water extract 1:2.5 v/v) were analyzed in the Desert Research Center laboratories according to Chapman and Pratt (1961).

\subsection{Fertilizer forms}

The fertilization treatments included the following 2.3.1. Chemical fertilizers

1-1-Control with recommended dose of N P K (F1) as (400kg per fed) ammonium sulphate $(20.5 \% \mathrm{~N})+$ phosphorus source at the rate of $300 \mathrm{~kg}$ calcium super-phosphate $\left(15.5 \% \mathrm{P}_{2} \mathrm{O}_{5}\right)$ per feddan + potassium source, at the rate of $100 \mathrm{~kg}$ potassium sulfate $\left(48 \% \quad \mathrm{~K}_{2} \mathrm{O}\right)$ per feddan plus compost added before planting 5 ton/fed.

1-2- ( $75 \%$ of the control recommended dose of $\mathrm{N}$ $\mathrm{P}$ K.( F2) as (300 kg per feddan) ammonium sulphate $(20.5 \% \mathrm{~N})+$ phosphorus, at the rate of $225 \mathrm{~kg}$ calcium super-phosphate $\left(15.5 \% \mathrm{P}_{2} \mathrm{O}_{5}\right)$ per feddan + potassium, at the rates of $75 \mathrm{~kg}$ potassium sulfate $\left(48 \% \quad \mathrm{~K}_{2} \mathrm{O}\right)$ per feddan plus compost added before planting 5 ton/fed.

1-3- F3( 50\% recommended dose)-The chemical fertilizers of N P K (F3) $200 \mathrm{~kg}$ per feddan ammonium sulphate $(20.5 \% \mathrm{~N})+$ phosphorus, at the rate of $150 \mathrm{~kg}$ calcium super-phosphate $(15.5$ $\% \mathrm{P}_{2} \mathrm{O}_{5}$ ) per feddan + potassium, at the rate of 50 $\mathrm{kg}$ potassium sulfate $\left(48 \% \mathrm{~K}_{2} \mathrm{O}\right)$ per feddan plus compost added before planting 5 ton/fed.

Fertilization with calcium super phosphate and compost 5 ton /fed. was conducted immediately before planting in each season in only one dose. As for nitrogen and potassium fertilizers they were applied in five equal doses in the both seasons. The first was added 21 days from appearance of the real leaves and then every two week intervals.

\subsubsection{Bio-fertilizer}

Biofertilizer was added as mixture of 5 strains of bacteria namely: Azotobacter chroococcum, Azospirillum lipoferum, Bacillus polymixa, B. megatherium and Pseudomonas fluorescence $\left(1 \mathrm{X} 10^{8} / \mathrm{ml}\right)$ obtained from Desert Research Center, El-Mataria, Egypt. The biofertilizer (1 
Table (1): Mechanical and chemical analyses of the soil.

\begin{tabular}{|c|c|c|c|c|c|c|c|c|c|c|c|}
\hline \multirow[t]{2}{*}{$\begin{array}{l}\text { Physical } \\
\text { analysis }\end{array}$} & $\begin{array}{c}\text { Very } \\
\text { coarse } \\
\text { sand\% }\end{array}$ & $\begin{array}{c}\text { Coarse sand } \\
\%\end{array}$ & $\begin{array}{c}\text { Medium } \\
\text { sand } \\
\%\end{array}$ & $\begin{array}{c}\text { Fine } \\
\text { sand \% }\end{array}$ & $\begin{array}{l}\text { Very } \\
\text { fine } \\
\text { sand }\end{array}$ & $\begin{array}{c}\text { Silt } \\
\text { and } \\
\text { clay } \\
\%\end{array}$ & \multicolumn{5}{|c|}{ Type of soil } \\
\hline & 0.31 & 1.93 & 40.05 & 53.52 & 2.44 & 1.75 & \multicolumn{5}{|c|}{ Sandy soil } \\
\hline \multirow{3}{*}{$\begin{array}{c}\text { Chemical } \\
\text { analysis }\end{array}$} & pH & E.C. & O.M. & \multicolumn{4}{|c|}{ Cations meq/L } & \multicolumn{4}{|c|}{ Anions meq/L } \\
\hline & 8.37 & 0.90 & 0.52 & $\mathrm{Ca}^{++}$ & $\mathbf{M g}^{++}$ & $\mathrm{Na}^{+}$ & $\mathbf{K}^{+}$ & $\mathrm{CO}_{3}^{-}$ & $\mathrm{HCO}_{3}^{-}$ & $\mathrm{Cl}^{-}$ & $\mathrm{SO}_{4}^{--}$ \\
\hline & & & & 4.08 & 0.82 & 4.50 & 0.25 & - & 1.91 & 3.00 & 4.74 \\
\hline
\end{tabular}

Table (2 ): Chemical analysis of the irrigation water.

\begin{tabular}{|l|c|c|c|c|c|c|c|c|c|c|}
\hline \multirow{2}{*}{$\begin{array}{l}\text { TDS } \\
\text { ppm }\end{array}$} & \multirow{2}{*}{ SH } & \multicolumn{4}{|c|}{ Soluble cations (ppm.) } & \multicolumn{4}{c|}{ Soluble anions (ppm.) } \\
\cline { 3 - 9 } & & $\mathrm{Ca}^{++}$ & $\mathrm{Mg}^{++}$ & $\mathrm{Na}^{+}$ & $\mathrm{K}^{+}$ & & $\mathbf{C O}^{-3}$ & $\mathbf{H C O}^{-3}$ & $\mathbf{S O}^{-4}$ & $\mathbf{C L}^{-1}$ \\
\hline 3355 & $\mathbf{8 . 1}$ & $\mathbf{2 1 5 . 6 8}$ & $\mathbf{1 2 8 . 6 4}$ & $\mathbf{7 2 0 . 0 0}$ & $\mathbf{8 . 0 0}$ & & $\mathbf{2 0 . 2 5}$ & $\mathbf{1 4 8 . 2 3}$ & $\mathbf{7 2 5 . 0}$ & $\mathbf{1 3 0 0 . 0}$ \\
& & & & & & & & & & \\
\hline
\end{tabular}


$\mathrm{L}$ mixture of 5 strains of bacteria $+20 \mathrm{~L}$ of water) was added as a soil drench four times per season at monthly intervals started from sowing the seeds. The addition of biofertilizer was done after one week from the addition of chemical fertilizers.

\subsubsection{Organic fertilizer}

Compost manure $(\mathrm{cm})$ was used at the rate of ( 5 ton per feddan) $(1 \mathrm{~m} 3=450-500 \mathrm{~kg}$ Compost $)$. The compost manure was obtained from El-obor market- El-obor city. The chemical characteristics of the compost are shown in Table (3). Common cultural practices were used throughout the experiment, including weed control which was similarly done whenever needed. Organic fertilizer was analyzed in the Desert Research Center laboratories.

\subsection{The treatments were conducted as follows}

F1 Control (100\%recommended dose)+ compost $(\mathrm{CM})$.

F2 (75\% recommended dose) + compost $(\mathrm{CM})$

F3 (50\% recommended dose) + compost (CM)

F4 Biofertilizer+5ton compost/fed.

F5 (100\% recommended dose) + compost $(\mathrm{CM})+$ biofertilizer

F6 (75\% recommended dose $)+\operatorname{compost}(\mathrm{CM})+$ biofertilizer

F7 (50\% recommended dose) + compost $(\mathrm{CM})+$ biofertilizer

\subsection{Harvesting}

Plants of fennel were harvested at the mature stage on $30^{\text {th }}$ April for $7^{\text {th }}$ Oct and $7^{\text {th }}$ May for both $21^{\text {st }}$ Oct and $7^{\text {th }}$ Nov in both seasons.

\subsection{Data recorded}

2.6.1. Vegetative characteristics at beginning of flowering

1.1. Plant height $(\mathrm{cm})$

1.2. Number of main branches per plant

1.3. Fresh and dry weights of herb (g/plant)

\subsubsection{Fruit measurements were recorded} at harvesting time

1. Umbels number/plant

2. Fruit yield /plant (g) and per feddan (ton)

3. Weight of 1000 fruits $(\mathrm{g})$

\subsubsection{Determination of essential oil production}

1. Essential oil \%

2. Essential oil yield per plant (ml) and per fed. (L)

3. Essential oil constituents by GC-Ms analysis:

Volatile oil percentage and content of dry fruits for each sample were determined using Clevenger apparatus according to (British Pharmacopoeia 1963). Also,GC/ Mass analysis of volatile oil of each treatment was performed with specification of the apparatus used according to Robert Adams
(1995).

\subsubsection{Element Determination ( $N, P$ and $K$ ) in} leaves

Nitrogen percentage was determined by modified micro kjeldahl method as described by A.O. A. C. (1970). For phosphorus determination, the ammonium molybdate method according to Murphy and Riley (1962) was used. As for potassium it was estimated used atomic absorption apparatus according to Cottenie et al. (1982).

\subsection{Statistical analysis}

The layout of the experiment was a complete randomized block design with three replicates (blocks), each replicate contained 10 plants. Data from all experiments were subjected to analysis of variance using $M$. statc Statistical Software (1986). Means of all data were compared by L.S.D. method according to (Snedecor and Cochran, 1968).

\section{RESULTS AND DISCUSSION}

\subsection{Vegetative characters}

Data in Tables (5\&6) indicated the main effect of fertilization treatments on vegetative parameters. The highest values of those parameters were obtained with the application of F5 (100\%recommended dose) + compost $(\mathrm{CM})+$ biofertilizer which produced the tallest plants, the highest number of branches/plants, the heaviest fresh and dry weights of herb/plant compared to other fertilization treatments resulting in significant differences in most cases. However, the highest rate of chemical fertilizer F1 Control (100\%recommended dose)+ compost(CM) gave the next higher values of vegetative parameters followed by F6 (75\%recommended dose) + compost $(\mathrm{CM})+$ biofertilizer). Meanwhile, fertilization treatment F4 (Biofertilizer+5ton compost/fed ) produced the lowest growth in the first and second seasons. Also, as sowing date became later up to $7^{\text {th }}$ November the fresh and dry weights showed a parallel significant decrease than in early sowing date ( $7^{\text {th }}$ October). The interaction between fertilization treatments and sowing dates was insignificant in most cases, however the highest vegetative parameters resulted from fertilization treatment F5 (100\% recommended dose) + compost $(\mathrm{CM})+$ biofertilizer at the early date of $7^{\text {th }}$ October. These results are coincided with those obtained by, Abd El-Latif and Salem (2002) on Tagetes minuta, L. and Rashed (2002) on Anethum graveolens, Coriandrum sativum and Petroselinum sativum plants. 
Table (3): The chemical properties of compost ( CM ). used for growing Foeniculum vulgare, Mill. plants during 2008 and 2009 seasons.

\begin{tabular}{|c|c|c|c|c|c|c|c|c|c|}
\hline \multirow{2}{*}{ pH } & $\begin{array}{c}\text { E.C } \\
\text { mmhos }\end{array}$ & \multicolumn{4}{|c|}{$\begin{array}{c}\text { Soluble cations } \\
\text { meq/L. }\end{array}$} & \multicolumn{4}{c|}{$\begin{array}{c}\text { Soluble anions } \\
\text { meq/L }\end{array}$} \\
\cline { 2 - 10 } & & $\mathrm{Ca}^{++}$ & $\mathrm{Mg}^{++}$ & $\mathrm{Na}^{+}$ & $\mathrm{K}^{+}$ & $\mathrm{CO}_{3}^{--}$ & $\mathrm{HCO}_{3}^{-}$ & $\mathrm{Cl}^{-}$ & $\mathrm{SO}_{4}^{--}$ \\
\hline 8.10 & 1.10 & 15.00 & 45.00 & 90.00 & 30.00 & - & 45.00 & 10.00 & 140.50 \\
\hline \multicolumn{2}{|c|}{ Humidity } & Ash & $\mathbf{O . M}$ & $\mathrm{N \%}$ & \multicolumn{2}{|c|}{$\mathbf{P \%}$} & $\mathrm{K} \%$ \\
\hline \multicolumn{2}{|c|}{$25 \%$} & $9 \%$ & $65 \%$ & $2 \%$ & \multicolumn{2}{|c|}{$1.5 \%$} & $1 \%$ \\
\hline
\end{tabular}

Organic fertilizer was analyzed in the Desert Research Center laboratories.

Table (4): Meteorological data of El-Sheikh Zwaid of North Sinai during 2008/2009 and 2009/2010 seasons.

\begin{tabular}{|c|c|c|c|c|c|c|c|c|}
\hline \multirow[t]{3}{*}{ Month } & \multicolumn{4}{|c|}{ First season } & \multicolumn{4}{|c|}{ Second season } \\
\hline & \multicolumn{2}{|c|}{ Temp. $\mathrm{C}^{\circ}$} & \multirow{2}{*}{$\begin{array}{c}\text { Relative } \\
\text { Humidity } \\
\%\end{array}$} & \multirow{2}{*}{$\begin{array}{c}\text { Rain } \\
\text { mm }\end{array}$} & \multicolumn{2}{|c|}{ Temp. C } & \multirow{2}{*}{$\begin{array}{c}\text { Relative } \\
\text { Humidity } \\
\%\end{array}$} & \multirow{2}{*}{$\begin{array}{c}\text { Rain } \\
\text { mm }\end{array}$} \\
\hline & $\max$. & min. & & & $\max$. & min. & & \\
\hline October & 28.1 & 15.5 & 70 & 5.50 & 24.2 & 13.5 & 70 & 20.50 \\
\hline November & 24.6 & 12.7 & 72 & 15.50 & 23.2 & 13.8 & 71 & 36.6 \\
\hline December & 21.5 & 10.2 & 81 & 19.50 & 18.2 & 7.5 & 73 & 138.4 \\
\hline January & 20.2 & 9.3 & 72 & 32.7 & 17.1 & 8.4 & 75 & 123.6 \\
\hline February & 20.4 & 8.3 & 66 & 23.9 & 17.7 & 6.0 & 70 & 117.4 \\
\hline March & 20.6 & 9.3 & 75 & 28.8 & 20.7 & 8.5 & 70 & 88.2 \\
\hline April & 27.1 & 13.6 & 60 & 0.7 & 26.2 & 11.2 & 64 & 23.6 \\
\hline May & 27.7 & 15.00 & 69 & 0.0 & 27.7 & 14.4 & 71 & 0.0 \\
\hline June & 35.7 & 22.00 & 79 & 0.0 & 36.1 & 24.00 & 84 & 0.0 \\
\hline July & 34.2 & 23.00 & 80 & 0.0 & 35.5 & 25.00 & 82 & 10.0 \\
\hline
\end{tabular}

* Meteorological Laboratory, Desert Research Center at El-Sheikh Zowaidd. 
Table (5): Effect of sowing dates and fertilization treatments on plant height (cm) and number of branches/plant of Foeniculum vulgare var panmorium (Indian fennel type) plants under North Sinai conditions during 2008/2009 and2009/2010 seasons.

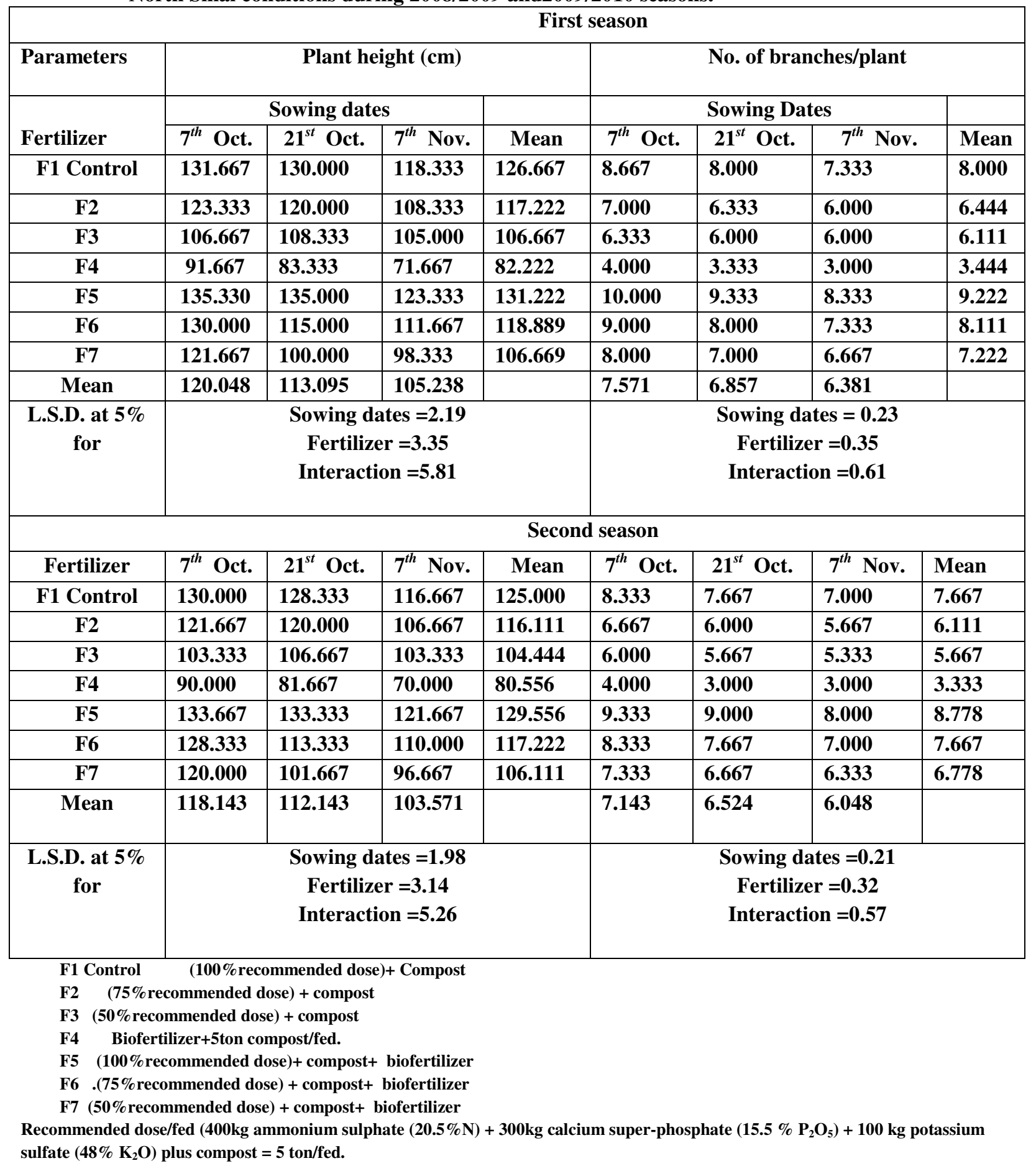


Table (6): Effect of sowing date and fertilization treatments on Fresh and dry weights of herb (g/plant) of Foeniculum vulgare var panmorium (Indian fennel type) plants under north Sinai conditions during 2008/2009 and2009/2010 seasons.

\begin{tabular}{|c|c|c|c|c|c|c|c|c|}
\hline & \multicolumn{8}{|c|}{ First season } \\
\hline Parameters & \multicolumn{4}{|c|}{ Fresh weight of herb (g/plant) } & \multicolumn{4}{|c|}{ Dry weight of herb (g/plant) } \\
\hline \multirow[b]{2}{*}{ Fertilizer } & \multicolumn{3}{|c|}{ Sowing Dates } & \multirow[b]{2}{*}{ Mean } & \multicolumn{3}{|c|}{ Sowing Dates } & \multirow[b]{2}{*}{ Mean } \\
\hline & $7^{\text {th }}$ Oct. & $21^{s t}$ Oct. & $7^{\text {th }}$ Nov. & & $7^{\text {th }}$ Oct. & $21^{s t}$ Oct. & $7^{\text {th }}$ Nov. & \\
\hline F1 Control & 723.333 & 700.000 & 616.667 & 680.000 & 122.357 & 117.610 & 109.797 & 116.588 \\
\hline F2 & 636.667 & 606.667 & 575.000 & 606.111 & 114.697 & 111.540 & 104.267 & 110.168 \\
\hline F3 & 553.333 & 510.00 & 500.00 & 521.111 & 96.397 & 91.123 & 92.133 & 93.218 \\
\hline F4 & 233.333 & 150.000 & 133.333 & $\mathbf{1 7 2 . 2 2 1}$ & 42.890 & 31.333 & 31.133 & 35.119 \\
\hline F5 & 833.333 & 800.000 & 733.333 & 788.889 & 144.463 & 138.000 & 128.800 & $\mathbf{1 3 7 . 0 8 8}$ \\
\hline F6 & 775.000 & 718.333 & 666.667 & 720.000 & 135.223 & 129.00 & 124.533 & 129.586 \\
\hline F7 & 593.333 & 540.000 & 433.333 & 522.222 & 120.717 & 93.587 & 74.000 & 96.101 \\
\hline Mean & 621.190 & 575.000 & 522.619 & & 110.963 & 101.742 & 94.952 & \\
\hline \multirow[t]{2}{*}{$\begin{array}{l}\text { L.S.D. at } 5 \% \\
\text { for }\end{array}$} & \multicolumn{4}{|c|}{$\begin{array}{l}\text { Sowing dates }=15.42 \\
\text { Interaction }=\mathbf{4 0 . 8 1}\end{array}$} & \multicolumn{4}{|c|}{$\begin{array}{l}\text { Sowing dates }=1.63 \\
\text { Fertilizer }=2.49 \\
\text { Interaction }=4.32\end{array}$} \\
\hline & \multicolumn{8}{|c|}{ Second season } \\
\hline Fertilizer & $7^{\text {th }}$ Oct. & $21^{\text {st }}$ Oct. & $7^{\text {th }}$ Nov. & Mean & $7^{\text {th }}$ Oct. & $21^{s t}$ Oct. & $7^{\text {th }}$ Nov. & Mean \\
\hline F1 Control & 713.333 & 683.333 & 600.000 & 665.556 & 115.673 & 114.300 & 103.133 & 111.036 \\
\hline F2 & 600.000 & 600.000 & 551.667 & 583.889 & 108.033 & $\mathbf{1 0 7 . 5 3 3}$ & 100.933 & 105.500 \\
\hline F3 & 516.667 & 500.000 & 500.000 & 505.556 & 91.067 & 87.800 & 83.800 & 87.556 \\
\hline F4 & 216.667 & 131.667 & 116.667 & 155.000 & 39.567 & 26.000 & 28.800 & 31.456 \\
\hline F5 & 816.667 & 783.333 & 703.333 & 767.778 & 136.467 & 124.667 & 122.133 & 127.756 \\
\hline F6 & 741.667 & 700.000 & 636.667 & 692.778 & 130.900 & 124.000 & 107.867 & 120.922 \\
\hline F7 & 576.667 & 516.667 & 416.667 & 503.333 & 114.700 & 88.600 & 69.000 & 90.767 \\
\hline Mean & 597.381 & 559.286 & 503.571 & & 105.201 & 96.129 & 87.952 & \\
\hline \multirow[t]{2}{*}{$\begin{array}{l}\text { L.S.D. at } 5 \% \\
\text { for }\end{array}$} & \multicolumn{4}{|c|}{$\begin{array}{ll}\text { Sowing dates }=14.13 & \text { Fertilizer }=21.42 \\
\text { Interaction }=36.67 & \end{array}$} & \multicolumn{4}{|c|}{$\begin{array}{l}\text { Sowing dates }=1.29 \\
\text { Fertilizer }=2.23 \text { Interaction }=3.93\end{array}$} \\
\hline & \multicolumn{8}{|c|}{$\begin{array}{ll}\text { F1 Control } & (100 \% \text { recommended dose })+\text { Compost }\end{array}$} \\
\hline $\begin{array}{lr}\text { F2 } & (\mathbf{7 5 \%} \\
\text { F3 } & (\mathbf{5 0 \%} \mathrm{r} \\
\text { F5 } & (\mathbf{1 0 0 \%} \\
\text { F6 } & . \mathbf{( 7 5 \%} \mathrm{r}\end{array}$ & $\begin{array}{l}\text { commended } \\
\text { ommended d } \\
\text { commended } \\
\text { ommended d }\end{array}$ & $\begin{array}{l}\text { lose) + compos } \\
\text { se) + compost } \\
\text { lose)+ compost } \\
\text { se) + compost }\end{array}$ & $\begin{array}{l}4 \quad \text { Bioferti } \\
\text { biofertilizer } \\
\text { biofertilizer }\end{array}$ & er+5ton con & post/fed. & & & \\
\hline
\end{tabular}
sulfate $(48 \% \mathrm{K2O})$ plus compost $=5$ ton/fed. 


\subsection{Fruit production}

\subsubsection{Number of umbels/plant}

Data in Table (7) showed that the highest number of umbels/plant was obtained from fertilization treatment F5 fertilizer $(100 \%$ recommended dose $)+$ compost $(\mathrm{CM})+$ (100\%recommended dose $)+$ compost $(\mathrm{CM})+$ biofertilizer, while F1 Control (100\% recommended dose $)+\operatorname{compost}(\mathrm{CM})$ and $\mathrm{F} 6$ (75\% recommended dose) $+\operatorname{compost}(\mathrm{CM})+$ biofertilizer resulted in significantly less number of umbels. The least significant number of umbels was obtained from fertilization treatment of F4 (Biofertilizer +5 ton compost/fed) which produced ( 15.33 and 16.00 umbels per plant) in the first and second seasons, respectively. Also delaying planting date to $7^{\text {th }}$ November caused a significant decrease in umbel number/plant. These results are coincided with those obtained by Ibrahem (2000) on Ammi visnaga L. and Foeniculum vulgare Mill. and Shalan et al. (2001) on roselle and Ayub et al. (2008) on fennel.

\subsubsection{Seed index (weight of 1000 seeds $(\mathrm{g})$ )}

Data in Table (7) show that the highest average of seed index (1000 seed weight) belonged to fertilization treatment F5, while the least average belonged to fertilization treatment $\mathrm{F} 4$ (Biofertilizer +5 ton compost/fed). Delaying sowing date to $7^{\text {th }}$ November resulted in a significant decrease in 1000 seed weight. The interaction between fertilization and sowing date was significant, however the highest 1000 seed weight belonged to fertilization treatment F5 $(100 \%$ recommended dose $)+$ compost $(\mathrm{CM})+$ biofertilizer) planted at the early date of $7^{\text {th }}$ October. Similar results were obtained by Osman (2000) on coriander and Gad (2001) on Foeniculum vulgare Mill and Anethum graveolens L.

\subsubsection{Fruit yield per plant and per feddan}

Data concerning the effect of different fertilization treatments on fruit yield per plant are presented in Table (8). These data cleared that the treatment of F5 fertilizer (100\%recommended dose $)+$ compost $(\mathrm{CM})+$ biofertilizer resulted in the highest fruit yield per plant. The other treatments significantly resulted in less fruit yield/plant being the least at F4 ( Biofertilizer+5 ton compost/fed.). Also, delaying sowing date to $7^{\text {th }}$ November resulted in the least significant yield of fruits/plant. The interaction between fertilizers and sowing date proved that the highest yield of fruits/plant was belonging to sown Indian fennel plants on $7^{\text {th }}$ of Oct. and fertilized with F5, while the lowest fruit yield /plant was obtained by treating the plants with F4 and planted in the $7^{\text {th }}$ of Nov. in both seasons.

Fruit yield per feddan in Table (8) shows a similar trend of fruit yield/plant where the highest yield/feddan was generally obtained by F5 with early sowing date of $7^{\text {th }}$ October, whereas the least yield was obtained by $\mathrm{F} 4$ at the delayed sowing date of $7^{\text {th }}$ November. Similar results were obtained by Badawi (2000) on roselle and Osman (2000) on coriander and Mahfouz and SharafEldin (2007) on fennel plants.

\subsection{Essential oil production}

\subsubsection{Oil percentage and oil yield/plant and/ feddan}

Data in Table (9) show that the differences between fertilization treatments were significant, where the highest oil percentage belonged to fertilization treatment F4 (Biofertilizer +5 ton compost/fed). However, the highest oil yield/plant and /fed was obtained from F5 fertilizer $(100 \%$ recommended dose $)+\operatorname{compost}(\mathrm{CM})+$ biofertilizer), whereas the least oil yield/plant and /fed was obtained from fertilization treatment F4. Also, delaying planting decreased both oil percentage and oil yield/plant and /fed. The highest values belonged to the early sowing date of $7^{\text {th }}$ October.

The interaction effects between fertilization treatments and sowing dates on oil percentage and oil yield were significant. The highest oil percentage in fruits were produced in the earliest date ( $7^{\text {th }}$ of Oct.) when the plants treated with F4 treatment. This may be due to the shortage of nutrition rates was sufficient to direct plants towards building up secondary metabolites including volatile oil.

The highest oil yield/plant and /fed belonged to F5 fertilizer, which planted in the $7^{\text {th }}$ of Oct. On the reverse, the least oil yield per plant and /fed produced from plants planted in the $7^{\text {th }}$ of Nov and treated with $\mathrm{F} 4$ treatment.

The increase in oil yield per plant may be due to the beneficial effects of these fertilizers on the synthesis of carbohydrates in plant tissues, consequently used for oil production. These results are in harmony with those found by $\mathrm{Al}-$ Humaid (2004) on fennel plants.

\subsubsection{Essential Oil constituents}

Analyzing the volatile oil of Indian fennel using G-C Mass spectrometry picnics revealed there were differences in the main components of the oil, according to sowing dates used in this investigation, as shown in Table (10) and chromatography Fig (1,2 and 3).

In the early date ( $7^{\text {th }}$ of Oct.) as shown in (Fig.1) 
Table (7): Effect of sowing date and fertilization treatments on umbel number per plant (g/ plant) and weight of 1000 fruits (g) of Foeniculum vulgare var panmorium (Indian fennel type) plants under north Sinai conditions during 2008/2009 and2009/2010 seasons.

\begin{tabular}{|c|c|c|c|c|c|c|c|c|}
\hline & \multicolumn{8}{|c|}{ First season } \\
\hline Parameters & \multicolumn{4}{|c|}{ Umbel number/ plant } & \multicolumn{4}{|c|}{ Weight of 1000 fruits (g) } \\
\hline \multirow[b]{2}{*}{ Fertilizer } & \multicolumn{3}{|c|}{ Sowing Dates } & & \multicolumn{3}{|c|}{ Sowing Dates } & \multirow[b]{2}{*}{ Mean } \\
\hline & $7^{\text {th }}$ Oct. & $21^{\text {st }}$ Oct. & $7^{\text {th }}$ Nov. & Mean & $7^{\text {th }}$ Oct. & $21^{s t}$ Oct. & $7^{\text {th }}$ Nov. & \\
\hline F1 Control & 40.333 & 37.667 & 31.667 & 36.556 & 5.117 & 5.017 & 5.003 & 5.046 \\
\hline F2 & 34.000 & 31.667 & 28.333 & 31.333 & 5.000 & 4.930 & 4.900 & 4.943 \\
\hline F3 & 29.667 & 25.667 & 20.000 & 25.111 & 4.173 & 4.020 & 4.053 & 4.082 \\
\hline F4 & 22.000 & 20.00 & 15.333 & 19.111 & 3.057 & 2.893 & 2.803 & 2.918 \\
\hline F5 & 46.000 & 42.667 & 36.667 & 41.778 & 5.680 & 5.360 & 5.070 & 5.370 \\
\hline F6 & 41.000 & 39.333 & 35.667 & 38.667 & 5.060 & 4.950 & 4.940 & 4.983 \\
\hline F7 & 34.000 & 31.333 & 29.667 & 31.667 & 4.257 & 4.070 & 4.043 & 4.123 \\
\hline Mean & 35.286 & 32.619 & 28.190 & & 4.620 & 4.463 & 4.402 & \\
\hline \multirow[t]{2}{*}{$\begin{array}{l}\text { L.S.D. at } \\
5 \% \text { for }\end{array}$} & \multicolumn{4}{|c|}{$\begin{array}{c}\text { Sowing dates }=1.10 \\
\text { ertilizer }=1.55 \text { Interaction }=2.69\end{array}$} & \multicolumn{4}{|c|}{$\begin{array}{c}\text { Sowing dates }=0.11 \\
\text { Fertilizer }=0.17 \text { Interaction }=0.29\end{array}$} \\
\hline & \multicolumn{8}{|c|}{ Second season } \\
\hline Fertilizer & $7^{\text {th }}$ Oct. & $21^{s t}$ Oct. & $7^{\text {th }}$ Nov. & Mean & $7^{\text {th }}$ Oct. & $21^{s t}$ Oct. & $7^{\text {th }}$ Nov. & Mean \\
\hline F1 Control & 38.333 & 36.000 & 30.667 & 35.000 & 5.100 & 4.673 & 5.017 & 4.930 \\
\hline F2 & 31.667 & 30.333 & 27.000 & 29.667 & 5.000 & 4.933 & 4.900 & 4.944 \\
\hline F3 & 27.667 & 25.000 & 19.333 & 24.000 & 4.167 & 4.007 & 4.067 & 4.080 \\
\hline F4 & 20.333 & 18.333 & 16.000 & 18.222 & 3.067 & 2.900 & 2.800 & 2.922 \\
\hline F5 & 42.667 & 40.333 & 35.000 & 39.333 & 5.700 & 5.433 & 5.100 & 5.411 \\
\hline F6 & 39.000 & 36.667 & 31.333 & 35.667 & 5.040 & 4.933 & 4.933 & 4.969 \\
\hline F7 & 30.667 & 30.000 & 27.333 & 29.333 & 4.233 & 4.043 & 4.033 & 4.103 \\
\hline Mean & 32.905 & 30.952 & 26.667 & & 4.615 & 4.418 & 4.407 & \\
\hline $\begin{array}{l}\text { L.S.D. at } \\
5 \% \text { for }\end{array}$ & \multicolumn{4}{|c|}{$\begin{array}{c}\text { Sowing dates }=\mathbf{1 . 0 4} \\
\text { Fertilizer }=\mathbf{1 . 4 4} \text { nteraction }=\mathbf{2 . 4 0}\end{array}$} & \multicolumn{4}{|c|}{$\begin{array}{l}\text { Sowing dates }=0.12 \\
\text { lizer }=0.19 \text { Interaction }=0.31\end{array}$} \\
\hline
\end{tabular}

F1 Control (100\% recommended dose)+ Compost

F2 (75\% recommended dose) + compost

F3 (50\% recommended dose) + compost F4 Biofertilizer+5ton compost/fed.

F5 (100\% recommended dose)+ compost+ biofertilizer

F6 . $.75 \%$ recommended dose $)+$ compost + biofertilizer

F7 (50\% recommended dose $)+$ compost + biofertilizer

Recommended dose/fed $\left(400 \mathrm{~kg}\right.$ ammonium sulphate $(20.5 \% \mathrm{~N})+300 \mathrm{~kg}$ calcium super-phosphate $\left(15.5 \% \mathrm{P}_{2} \mathrm{O}_{5}\right)+100 \mathrm{~kg}$ potassium sulfate $\left(48 \% \mathrm{~K}_{2} \mathrm{O}\right)$ plus compost $=5$ ton/fed. 
Table (8): Effect of sowing date and fertilization treatments on fruit yield/plant (g) and fruit yield/feddan (ton) of Foeniculum vulgare subsp. Panmorium (Indian fennel type) plants under north Sinai conditions during 2008/2009 and2009/2010 seasons.

\begin{tabular}{|c|c|c|c|c|c|c|c|c|}
\hline \multicolumn{9}{|c|}{ First season } \\
\hline Parameters & \multicolumn{4}{|c|}{ Fruit yield / plant (g) } & \multicolumn{4}{|c|}{ Fruit yield per feddan (ton) } \\
\hline \multirow[b]{2}{*}{ Fertilizer } & \multicolumn{3}{|c|}{ Sowing Dates } & & \multicolumn{3}{|c|}{ Sowing Dates } & \multirow[b]{2}{*}{ Mean } \\
\hline & $7^{\text {th }}$ Oct. & $21^{\text {st }}$ Oct. & $7^{\text {th }}$ Nov. & Mean & $7^{\text {th }}$ Oct. & $21^{\text {st }}$ Oct. & $7^{\text {th }}$ Nov. & \\
\hline F1 Control & 66.149 & 63.149 & 59.149 & 62.816 & 1.482 & 1.415 & 1.325 & 1.407 \\
\hline F2 & 60.179 & 55.519 & 52.519 & 56.072 & 1.348 & 1.244 & 1.176 & 1.256 \\
\hline F3 & 51.179 & 49.519 & 27.509 & 42.736 & 1.146 & 1.109 & 0.616 & 0.957 \\
\hline F4 & 8.519 & 7.179 & 5.509 & 7.069 & 0.191 & 0.161 & 0.123 & 0.158 \\
\hline F5 & 75.519 & 72.519 & 70.149 & 72.729 & 1.692 & 1.624 & 1.571 & 1.629 \\
\hline F6 & 67.509 & 62.149 & 60.519 & 63.392 & 1.512 & 1.392 & 1.356 & 1.420 \\
\hline F7 & 31.179 & 30.509 & 23.519 & 28.402 & 0.698 & 0.683 & 0.527 & 0.636 \\
\hline Mean & 51.462 & 48.649 & 42.696 & & 1.153 & 1.090 & 0.956 & \\
\hline L.S.D. at $5 \%$ for & \multicolumn{4}{|c|}{$\begin{array}{c}\text { sowing dates }=4.87 \\
\text { fertilizer }=7.50 \\
\text { interaction }=14.18\end{array}$} & \multicolumn{4}{|c|}{ Values calculated } \\
\hline \multicolumn{9}{|c|}{ Second season } \\
\hline Fertilizer & $7^{\text {th }}$ Oct. & $21^{\text {st }}$ Oct. & $7^{\text {th }}$ Nov. & Mean & $7^{\text {th }}$ Oct. & $21^{\text {st }}$ Oct. & $7^{\text {th }}$ Nov. & Mean \\
\hline F1 Control & 64.62 & 61.64 & 57.66 & 61.31 & 1.45 & 1.38 & 1.29 & 1.37 \\
\hline F2 & 58.65 & 54.06 & 51.29 & 54.67 & 1.310 & 1.210 & 1.150 & 1.220 \\
\hline F3 & 49.65 & 48.05 & 26.38 & 41.36 & 1.110 & 1.080 & 0.590 & 0.930 \\
\hline F4 & 7.39 & 5.65 & 4.28 & 5.78 & 0.170 & 0.130 & 0.100 & 0.130 \\
\hline F5 & 74.09 & 71.29 & 68.62 & 71.34 & 1.660 & 1.600 & 1.540 & 1.600 \\
\hline F6 & 66.03 & 60.62 & 59.39 & 62.02 & 1.480 & 1.360 & 1.330 & 1.390 \\
\hline F7 & 29.65 & 29.08 & 22.19 & 26.98 & 0.660 & 0.650 & 0.500 & 0.600 \\
\hline Mean & 50.02 & 47.20 & 41.41 & & 1.120 & 1.060 & 0.930 & \\
\hline L.S.D. at $5 \%$ for & \multicolumn{4}{|c|}{$\begin{array}{c}\text { sowing dates }=4.16 \\
\text { fertilizer }=7.82 \\
\text { interaction }=13.96\end{array}$} & \multicolumn{4}{|c|}{ Values calculated } \\
\hline
\end{tabular}

F1 Control (100\% recommended dose)+ compost

F2 (75\% recommended dose) + compost

F3 $(50 \%$ recommended dose $)+$ compost

F4 Biofertilizer+5ton compost/fed.

F5 (100\% recommended dose)+ compost+ biofertilizer

F6 .(75\% recommended dose) + compost+ biofertilizer

F7 (50\% recommended dose) + compost + biofertilizer

Recommended dose/fed (400kg ammonium sulphate $(20.5 \% \mathrm{~N})+300 \mathrm{~kg}$ calcium super-phosphate $\left(15.5 \% \mathrm{P}_{2} \mathrm{O}_{5}\right)+100 \mathrm{~kg}$ potassium sulfate $\left(48 \% \mathrm{~K}_{2} \mathrm{O}\right)$ plus compost $=5$ ton/fed. 
Table (9): Effect of sowing date and fertilization treatments on essential oil percentage ,oil yield per plant (ml/ plant) and oil yield / feddan (liter) of Foeniculum vulgare var panmorium (Indian fennel type) plants under north Sinai conditions during 2008/2009 and2009/2010 seasons.

\begin{tabular}{|c|c|c|c|c|c|c|c|c|c|c|c|c|}
\hline \multicolumn{13}{|c|}{ First season } \\
\hline Parameters & \multicolumn{4}{|c|}{ Essential oil percentage } & \multicolumn{4}{|c|}{ Oil yield / plant (ml.) } & \multicolumn{4}{|c|}{ Oil yield / feddan (liter) } \\
\hline \multirow[b]{2}{*}{ Fertilizer } & \multicolumn{3}{|c|}{ Sowing Dates } & \multirow[b]{2}{*}{ Mean } & \multicolumn{3}{|c|}{ Sowing Dates } & \multirow[b]{2}{*}{ Mean } & \multicolumn{3}{|c|}{ Sowing Dates } & \multirow[b]{2}{*}{ Mean } \\
\hline & $\begin{array}{c}7^{\text {th }} \\
\text { Oct. }\end{array}$ & $\begin{array}{l}21^{s t} \\
\text { Oct. }\end{array}$ & $\begin{array}{c}7^{\text {th }} \\
\text { Nov. }\end{array}$ & & $\begin{array}{c}7^{\text {th }} \\
\text { Oct. }\end{array}$ & $\begin{array}{l}21^{s t} \\
\text { Oct. }\end{array}$ & $\begin{array}{c}7^{\text {th }} \\
\text { Nov. }\end{array}$ & & $\begin{array}{l}7^{\text {th }} \\
\text { Oct. }\end{array}$ & $\begin{array}{l}21^{s t} \\
\text { Oct. }\end{array}$ & $\begin{array}{c}7^{t h} \\
\text { Nov. }\end{array}$ & \\
\hline F1 cont. & 2.130 & 2.113 & 2.100 & 2.114 & 1.409 & 1.334 & 1.242 & 1.328 & 31.562 & 29.882 & 27.821 & 29.747 \\
\hline F2 & 2.127 & 2.107 & 2.097 & 2.110 & 1.280 & 1.170 & 1.101 & 1.183 & 28.672 & 26.208 & 24.662 & 26.499 \\
\hline $\mathbf{F 3}$ & 2.133 & 2.103 & 2.107 & 2.114 & 1.092 & 1.041 & 0.580 & 0.903 & 24.461 & 23.318 & 12.992 & 20.227 \\
\hline F4 & 2.270 & 2.250 & 2.237 & 2.252 & 0.193 & 0.161 & 0.123 & 0.159 & 4.323 & 3.606 & 2.755 & 3.562 \\
\hline F5 & 2.127 & 2.117 & 2.067 & 2.103 & 1.606 & 1.535 & 1.450 & 1.529 & 35.974 & 34.384 & 32.480 & 34.250 \\
\hline F6 & 2.140 & 2.123 & 2.100 & 2.121 & 1.445 & 1.319 & 1.271 & 1.344 & 32.368 & 29.546 & 28.470 & 30.106 \\
\hline F7 & 2.110 & 2.100 & 2.090 & 2.100 & 0.658 & 0.641 & 0.491 & 0.596 & 14.739 & 14.358 & 10.998 & 13.350 \\
\hline Mean & 2.148 & 2.130 & 2.114 & & 1.105 & 1.036 & 0.902 & & 24.752 & 23.206 & 20.205 & \\
\hline $\begin{array}{l}\text { L.S.D. at } \\
5 \% \text { for }\end{array}$ & \multicolumn{4}{|c|}{$\begin{array}{l}\text { Sowing dates }=0.019 \\
\text { Fertilizer }=\mathbf{0 . 0 2 8} \\
\text { Interaction }=\mathbf{0 . 0 4 9}\end{array}$} & \multicolumn{4}{|c|}{$\begin{array}{l}\text { Sowing dates }=0.048 \\
\text { Fertilizer }=0.074 \\
\text { Interaction }=\mathbf{0 . 1 2 8}\end{array}$} & \multicolumn{4}{|c|}{ Values calculated } \\
\hline \multicolumn{13}{|c|}{ Second season } \\
\hline Fertilizer & $\begin{array}{c}7^{\text {th }} \\
\text { Oct. }\end{array}$ & $\begin{array}{l}21^{s t} \\
\text { Oct. }\end{array}$ & $\begin{array}{c}7^{t h} \\
\text { Nov. }\end{array}$ & Mean & $\begin{array}{c}7^{\text {th }} \\
\text { Oct. }\end{array}$ & $\begin{array}{l}21^{s t} \\
\text { Oct. }\end{array}$ & $\begin{array}{c}7^{t h} \\
\text { Nov. }\end{array}$ & Mean & $\begin{array}{l}7^{\text {th }} \\
\text { Oct. }\end{array}$ & $\begin{array}{l}21^{s t} \\
\text { Oct. }\end{array}$ & $\begin{array}{l}7^{\text {th }} \\
\text { Nov. }\end{array}$ & Mean \\
\hline F1Cont. & 2.133 & 2.113 & 2.100 & 2.116 & 1.378 & 1.302 & 1.211 & 1.297 & 30.867 & 29.165 & 27.126 & 29.053 \\
\hline F2 & 2.133 & 2.107 & 2.103 & 2.114 & 1.251 & 1.139 & 1.079 & 1.156 & 28.022 & 25.514 & 24.170 & 25.894 \\
\hline F3 & 2.140 & 2.103 & 2.113 & 2.119 & 1.062 & 1.010 & $\mathbf{0 . 5 5 7}$ & 0.876 & 23.789 & 22.624 & 12.477 & 19.622 \\
\hline F4 & 2.263 & 2.253 & 2.233 & 2.250 & 0.167 & 0.127 & 0.095 & 0.130 & 3.741 & 2.845 & 2.128 & 2.912 \\
\hline F5 & 2.133 & 2.103 & 2.090 & 2.109 & 1.580 & 1.499 & 1.434 & 1.505 & 35.392 & 33.578 & 32.122 & 33.712 \\
\hline F6 & 2.140 & 2.123 & 2.100 & 2.121 & 1.413 & 1.287 & 1.247 & 1.315 & 31.651 & 28.829 & 27.933 & 29.456 \\
\hline F7 & 2.110 & 2.100 & 2.103 & 2.104 & 0.626 & 0.611 & 0.467 & 0.568 & 14.022 & 13.686 & 10.461 & 12.723 \\
\hline Mean & 2.150 & 2.129 & 2.120 & & 1.075 & 1.005 & 0.878 & & 24.080 & 22.512 & 19.667 & \\
\hline $\begin{array}{l}\text { L.S.D. at } \\
5 \% \text { for }\end{array}$ & \multicolumn{4}{|c|}{$\begin{array}{l}\text { Sowing dates }=\mathbf{0 . 0 1 8} \\
\text { Fertilizer }=\mathbf{0 . 0 2 7} \\
\text { Interaction }=\mathbf{0 . 0 4 8}\end{array}$} & $\begin{array}{l}\text { L.S.D. } \\
\text { at } 5 \% \\
\text { for }\end{array}$ & \multicolumn{3}{|c|}{$\begin{array}{l}\text { Sowing dates }=\mathbf{0 . 0 5 1} \\
\text { Fertilizer }=\mathbf{0 . 0 7 7} \\
\text { Interaction }=\mathbf{0 . 1 3 1}\end{array}$} & $\begin{array}{l}\text { L.S.D. } \\
\text { at } 5 \% \\
\text { for }\end{array}$ & \multicolumn{3}{|c|}{ Values calculated } \\
\hline
\end{tabular}

F1 Control $\quad(100 \%$ recommended dose $)+$ Compost

F2 (75\% recommended dose) + compost F3 (50\% recommended dose) + compost

F4 Biofertilizer+5ton compost/fed. F5 (100\% recommended dose $)+$ compost+ biofertilizer

F6 .(75\% recommended dose) + compost+ biofertilizer F7 (50\% recommended dose) + compost+ biofertilizer

Recommended dose/fed $\left(400 \mathrm{~kg}\right.$ ammonium sulphate $(20.5 \% \mathrm{~N})+300 \mathrm{~kg}$ calcium super-phosphate $\left(15.5 \% \mathrm{P}_{2} \mathrm{O}_{5}\right)+100$ $\mathrm{kg}$ potassium sulfate $\left(48 \% \mathrm{~K}_{2} \mathrm{O}\right)$ plus compost $=5$ ton/fed. 
1) the main component detected was transanethole $(54.15 \%)$ which is considered the important constituent, followed by fenchone $(14.88 \%)$ then estragole $(14.72 \%)$. There are other major components such as limonene $(8.81 \%)$ and , $\alpha$-pinene $(2.14 \%)$ found in the volatile oil.

Referring to the oil of the fruits sown in the middle date $21^{s t}$ of Oct. illustrated in Table (10) and chromatography (Fig 2), it contained transanethole as the main component $(52.31 \%)$. This value was less than that of the first date $\left(7^{\text {th }}\right.$ of Oct.) followed descendingly by estragole $(17.84 \%)$ then fenchone (14.91\%). Moreover, there are some components in small quantities such as limonene $(8.11 \%)$ and $\alpha$-pinene $(2.18 \%)$ which are considered important constituents.

Concerning the main component in the fruits of the plants planted in the $7^{\text {th }}$ of Nov and illustrated in Table (10) and Fig (3) it was trans-anethole. Its percentage was less $(46.11 \%)$ followed descendingly by estragole $(22.64 \%)$ followed descendingly by fenchone $(15.21 \%)$ then limonene $(8.14 \%)$ and $\alpha$-pinene $(2.21 \%)$. sowing date. Methyl-chavicol (estragole) was high in delayed dates, while limonene and $\rho$-cymene recorded the least values with late sowing date of 21 November. Razan (2010) sowed anise seeds on three dates $\left(15^{\text {th }}\right.$ Nov., $1^{\text {st }}$ Jan., $15^{\text {th }}$ Feb. $)$. He found that the best sowing date for anethole percentage was on $15^{\text {th }}$ Nov. which gave (91.83 $\%)$. Naguib et al. (2007) on Ruta graveolens L. showed that coumarin and rutin contents were higher with delaying sowing dates.

\subsubsection{Chemical composition (NPK)}

Data presented in Table (11) showed that, fertilized Indian fennel plants with F5 treatment showed its superiority for increasing nitrogen, phosphorus and potassium percentages in their tissues, followed descendingly by using F1 control. The positive effect of NPK fertilization on nitrogen percentage in Indian fennel might be due to more absorption and accumulation of nitrogen.

Concerning the specific effect of sowing dates on nitrogen, phosphorus and potassium (\%), the results recorded in Table (11) indicate that, the percentages of nitrogen, phosphorus and

Table (10): Effect of sowing date on essential oil constituents of Foeniculum vulgare subsp. panmorium (Indian fennel type) under North Sinai conditions.

\begin{tabular}{|c|c|c|c|c|}
\hline \multirow[t]{2}{*}{ Name of compound } & \multirow{2}{*}{$\begin{array}{c}\text { Retention } \\
\text { time }\end{array}$} & \multicolumn{3}{|c|}{ Area \% } \\
\hline & & $7^{\text {th }}$ Oct. & $21^{\text {th }}$ Oct. & $7^{\text {th }}$ Nov. \\
\hline 1.a-Pinene & 15.06 & 2.14 & 2.18 & 2.21 \\
\hline 2.Camphene & 15.37 & 0.63 & 0.65 & 0.66 \\
\hline 3.Sabinene & 16.93 & 0.21 & 0.20 & 0.18 \\
\hline 4. $\beta$-Pinene & 17.65 & $\mathbf{0 . 1 0}$ & 0.11 & 0.13 \\
\hline 5.Myrcene & 17.90 & $\mathbf{0 . 0 7}$ & 0.1 & 0.12 \\
\hline 6.Phellandrene & 18.03 & $\mathbf{0 . 2 0}$ & $\mathbf{0 . 2 0}$ & 0.25 \\
\hline 7.Limonene & 18.92 & 8.81 & 8.11 & 8.14 \\
\hline 8.Fenchone & 21.14 & 14.88 & 14.91 & 15.21 \\
\hline 9.Camphor & 22.60 & 0.05 & 0.06 & 0.06 \\
\hline 10.Estragole & 24.51 & 14.72 & 17.84 & 22.64 \\
\hline 11.Fenchyl acetate & 26.05 & 0.30 & 0.29 & 0.31 \\
\hline 12.Trans-anethole & 27.76 & 54.15 & 52.31 & 46.11 \\
\hline Other components & & 3.74 & 3.04 & 3.78 \\
\hline
\end{tabular}

These results coincided with those obtained by Jacoub (1995) on sweet basil (Ocimum basilicum) plants. He found that, chemical fertilizers with different rates caused insignificant effect on oil percentage, whereas, oil yield/plant and linalool content were increased., while methyl chavicol percentage was decreased. Abd El- Wahab and Mehasen (2009) on fennel found that delaying sowing date decreased anethol content in the volatile oil. Fenchone percentage in the fennel volatile oil was generally increased with delaying potassium in the leaves generally decreased by delaying the planting date from $7^{\text {th }}$ of Oct to $7^{\text {th }}$ of Nov in both seasons.

Referring to the interaction effect between fertilizers and sowing date data presented in Table (11) cleared that, the highest values of nitrogen, phosphorus and potassium percentages were recorded with the planting dates of $7^{\text {th }}$ of Oct, $21^{s t}$ of Oct and the $7^{\text {th }}$ of Nov respectively when the plants of Indian fennel were treated with F5 in both seasons. This increment may be due to the 


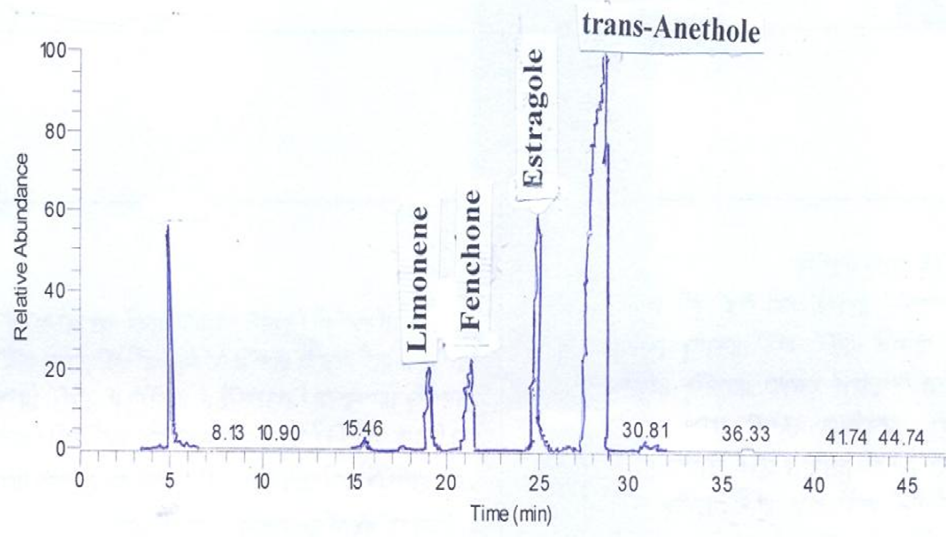

(Fig. 1): Effect of sowing date $7^{\text {th }}$ Oct. on Essential oil constituents of Foeniculum vulgare var. panmorium (Indian fennel type) plants under north Sinai conditions.

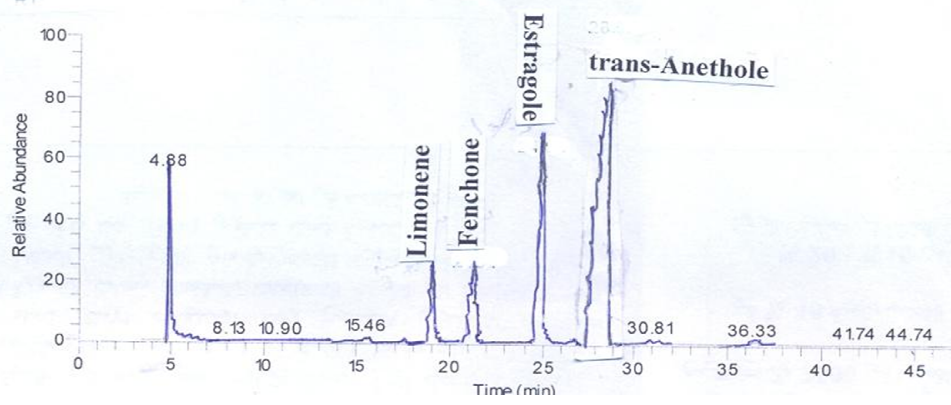

(Fig. 2): Effect of sowing date $21^{\text {st }}$ Oct. on Essential oil constituents of Foeniculum vilgare vat. panmorium (Indian fennel type) plants under north Sinai conditions.

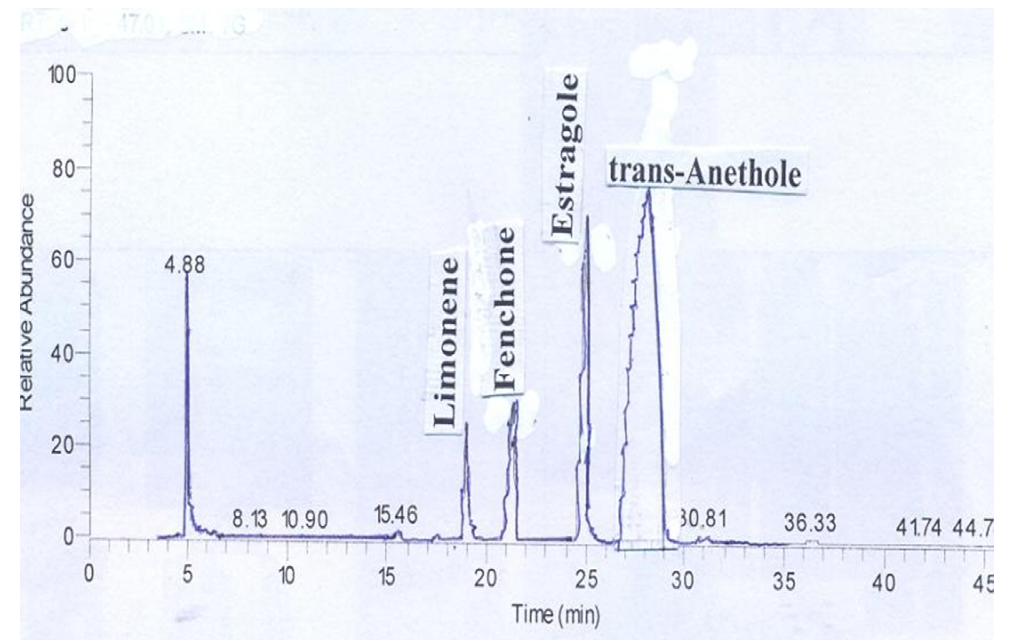

(Fig. 3): Effect of sowing date $7^{\text {th }}$ Nov. on Essential oil constituents of Foeniculum vulgare var: panmorium (Indian fennel type) plants under north Sinai conditions. 
Table (11): Effect of sowing date and fertilization treatments on nitrogen, phosphorus and potassium percentages of Foeniculum vulgare var. panmorium (Indian fennel type) plants under North Sinai conditions during 2008/2009 and2009/2010 seasons.

\begin{tabular}{|c|c|c|c|c|c|c|c|c|c|c|c|c|}
\hline \multicolumn{13}{|c|}{ First season } \\
\hline Parameters & \multicolumn{4}{|c|}{ Nitrogen $\%$} & \multicolumn{4}{|c|}{ Phosphorus \% } & \multicolumn{4}{|c|}{ Potassium \% } \\
\hline \multirow[b]{2}{*}{ Fertilizer } & \multicolumn{3}{|c|}{ Sowing Dates } & \multirow[b]{2}{*}{ Mean } & \multicolumn{3}{|c|}{ Sowing Dates } & \multirow[b]{2}{*}{ Mean } & \multicolumn{3}{|c|}{ Sowing Dates } & \multirow[b]{2}{*}{ Mean } \\
\hline & $\begin{array}{c}7^{\text {th }} \\
\text { Oct. }\end{array}$ & $\begin{array}{l}21^{s t} \\
\text { Oct. }\end{array}$ & $\begin{array}{c}7^{t h} \\
\text { Nov. }\end{array}$ & & $\begin{array}{l}7^{\text {th }} \\
\text { Oct. }\end{array}$ & $\begin{array}{l}21^{s t} \\
\text { Oct. }\end{array}$ & $\begin{array}{c}7^{t h} \\
\text { Nov. }\end{array}$ & & $\begin{array}{l}7^{\text {th }} \\
\text { Oct. }\end{array}$ & $\begin{array}{l}21^{s t} \\
\text { Oct. }\end{array}$ & $\begin{array}{c}7^{t h} \\
\text { Nov. }\end{array}$ & \\
\hline F1 Control & 1.560 & 1.540 & 1.500 & 1.533 & 0.360 & 0.340 & 0.310 & 0.337 & 3.360 & 3.340 & 3.320 & 3.340 \\
\hline F2 & 1.440 & 1.450 & 1.390 & 1.427 & 0.340 & 0.330 & 0.300 & 0.328 & 3.240 & 3.250 & 3.220 & 3.237 \\
\hline F3 & 1.310 & 1.290 & 1.270 & 1.290 & 0.310 & 0.290 & 0.270 & 0.290 & 3.220 & 3.210 & 3.200 & 3.210 \\
\hline F4 & 1.210 & 1.230 & 1.190 & 1.210 & 0.290 & 0.270 & 0.290 & 0.283 & 3.110 & 3.130 & 3.120 & 3.120 \\
\hline F5 & 1.640 & 1.630 & 1.580 & 1.617 & 0.440 & 0.380 & 0.360 & 0.393 & 3.540 & 3.530 & 3.480 & 3.517 \\
\hline F6 & 1.510 & 1.540 & 1.470 & 1.507 & 0.330 & 0.340 & 0.320 & 0.330 & 3.330 & 3.340 & 3.310 & 3.327 \\
\hline F7 & 1.340 & 1.320 & 1.290 & 1.317 & 0.300 & 0.320 & 0.290 & 0.303 & 3.140 & 3.120 & 3.120 & 3.127 \\
\hline Mean & 1.430 & 1.429 & 1.384 & & 0.339 & 0.324 & 0.306 & & 3.277 & 3.274 & 3.253 & \\
\hline $\begin{array}{l}\text { L.S.D. at } \\
5 \% \text { for }\end{array}$ & \multicolumn{4}{|c|}{$\begin{array}{c}\text { Sowing dates }=\mathbf{0 . 0 1 1} \\
\text { Fertilizer }=\mathbf{0 . 2 5} \\
\text { Interaction }=\mathbf{0 . 1 4 8}\end{array}$} & \multicolumn{4}{|c|}{$\begin{array}{c}\text { Sowing dates }=\mathbf{0 . 0 2 1} \\
\text { Fertilizer }=\mathbf{0 . 0 8 9} \\
\text { Interaction }=\mathbf{0 . 1 2 1}\end{array}$} & \multicolumn{4}{|c|}{$\begin{array}{c}\text { Sowing dates }=\mathbf{0 . 0 1 4} \\
\text { Fertilizer }=\mathbf{0 . 2 2 1} \\
\text { Interaction }=\mathbf{0 . 2 1 3}\end{array}$} \\
\hline \multicolumn{13}{|c|}{ Second season } \\
\hline Fertilizer & $\begin{array}{c}7^{\text {th }} \\
\text { Oct. }\end{array}$ & $\begin{array}{l}21^{s t} \\
\text { Oct. }\end{array}$ & $\begin{array}{c}7^{\text {th }} \\
\text { Nov. }\end{array}$ & Mean & $\begin{array}{c}7^{\text {th }} \\
\text { Oct. }\end{array}$ & $\begin{array}{l}21^{s t} \\
\text { Oct. }\end{array}$ & $\begin{array}{c}7^{t h} \\
\text { Nov. }\end{array}$ & mean & $\begin{array}{l}7^{\text {th }} \\
\text { Oct. }\end{array}$ & $\begin{array}{l}21^{s t} \\
\text { Oct. }\end{array}$ & $\begin{array}{l}7^{\text {th }} \\
\text { Nov. }\end{array}$ & Mean \\
\hline F1 Control & 1.580 & 1.560 & 1.490 & 1.543 & 0.360 & 0.330 & 0.310 & 0.333 & 3.380 & 3.360 & 3.350 & 3.363 \\
\hline F2 & 1.420 & 1.420 & 1.360 & 1.400 & 0.330 & 0.320 & 0.300 & 0.317 & 3.340 & 3.320 & 3.290 & 3.317 \\
\hline F3 & 1.340 & 1.310 & 1.290 & 1.313 & 0.320 & 0.300 & 0.280 & 0.300 & 3.260 & 3.250 & 3.260 & 3.257 \\
\hline F4 & 1.230 & 1.220 & 1.200 & 1.217 & 0.300 & 0.260 & 0.290 & 0.283 & 3.210 & 3.190 & 3.180 & 3.193 \\
\hline F5 & 1.630 & 1.580 & 1.530 & 1.580 & 0.420 & 0.370 & 0.350 & 0.380 & 3.510 & 3.510 & 3.490 & 3.503 \\
\hline F6 & 1.490 & 1.520 & 1.480 & 1.497 & 0.310 & 0.340 & 0.310 & 0.320 & 3.310 & 3.320 & 3.290 & 3.307 \\
\hline F7 & 1.320 & 1.300 & 1.300 & 1.307 & 0.300 & 0.310 & 0.290 & 0.300 & 3.230 & 3.200 & 3.190 & 3.207 \\
\hline Mean & 1.430 & 1.416 & 1.379 & & 0.334 & 0.319 & 0.304 & & 3.320 & 3.307 & 3.293 & \\
\hline $\begin{array}{l}\text { L.S.D. at } \\
5 \% \text { for }\end{array}$ & \multicolumn{4}{|c|}{$\begin{array}{c}\text { Sowing dates }=\mathbf{0 . 0 1 3} \\
\text { Fertilizer }=\mathbf{0 . 2 6} \\
\text { Interaction }=\mathbf{0 . 1 5 1}\end{array}$} & \multicolumn{4}{|c|}{$\begin{array}{c}\text { Sowing dates }=\mathbf{0 . 0 2 2} \\
\text { Fertilizer }=\mathbf{0 . 0 9 1} \\
\text { Interaction }=\mathbf{0 . 1 2 6}\end{array}$} & \multicolumn{4}{|c|}{$\begin{array}{c}\text { Sowing dates }=0.16 \\
\text { Fertilizer }=\mathbf{0 . 2 3 5} \\
\text { Interaction }=\mathbf{0 . 2 6 1}\end{array}$} \\
\hline
\end{tabular}

F1 Control (100\% recommended dose)+ Compost $\quad$ F2 (75\% recommended dose) + compost

F3 (50\% recommended dose) + compost F4 Biofertilizer+5ton compost/fed.

F5 (100\% recommended dose $)+$ compost+ biofertilizer F6 .(75\% recommended dose $)+$ compost+ biofertilizer

F7 $(50 \%$ recommended dose $)+$ compost + biofertilizer

Recommended dose/fed (400kg ammonium sulphate $(20.5 \% \mathrm{~N})+300 \mathrm{~kg}$ calcium super-phosphate $\left(15.5 \% \mathrm{P}_{2} \mathrm{O}_{5}\right)+100 \mathrm{~kg}$ potassium sulfate $(48 \%$ $\mathrm{K}_{2} \mathrm{O}$ ) plus compost $=5$ ton/fed. 
availability of nitrogen for plant absorption.

These results are in harmony with those reported by Badawi (2000) on roselle who found that, the highest increases of nitrogen, phosphorus and potassium in the leaves, seeds and sepals were recorded for the treatment of $(300 \mathrm{~kg} \mathrm{~N}+200 \mathrm{~kg}$ $\mathrm{P}+100 \mathrm{~kg} \mathrm{~K}+$ biofertilizers). Hamed (2004) on chamomile plants, mentioned that the highest values of nitrogen, phosphorus and potassium in the herb were recorded with the treatment of biofertilizer plus full dose of $\mathrm{N}$ and $\mathrm{P}$ than the other treatments and control. Abd El-Latif (2006) on Salvia officinalis, found that using poultry manure (PM) at $20 \mathrm{~m}^{3} / \mathrm{fed}$. increased nitrogen, phosphorus and potassium percentages in most cuts. Bishr et al., (2006) on borage (Borago officinalis), cleared that the treatment of chicken manure $\left(10 \mathrm{~m}^{3} / \mathrm{fed}.\right), \mathrm{Mn}(100 \mathrm{ppm})$ and their combination recorded the highest values in nitrogen, phosphorus and potassium /plant. These results are in harmony with those found by ElGhadban et al. (2008) on lavander (Lavendula multifida L.), who concluded that the effect of organic fertilizer (compost) at the rate of 25, 50 and $75 \mathrm{~g} /$ pot on chemical composition. The results revealed that increasing in $\mathrm{N}, \mathrm{P}$ and $\mathrm{K}$ percentages.

\section{REFERENCES}

Abd El-Latif E.S.M. (2006). Effect of chemical, organic fertilizers and spraying with active dry yeast on growth, oil production and plant constituents of sage (Salvia officinalis, L.) plant. M.Sc. Thesis, Fac. Agric., Cairo Univ., Egypt.

Abd El-Latif T.A. and Salem A. G. (2002). Influence of biofertilizer and NPK rate on Tagetes minuta, L. J. Agric. Sci. Mansoura Univ., 27 (4): 2475-2487.

Abd El- Wahab M.A. and Mehasen H.R.A (2009). Effect of locations and sowing date on (Foeniculum vulgare var. panmorium.) Indian fennel type under upper Egypt conditions. Journal of Applied Sciences Research, 5(6): 677-685, 2009

Abou Zeid E.N. (1988). Aromatic Plants. (In Arabic) El-Dar El-Arabia for Publication, Cairo.

Al-Humaid A.L. (2004). Effect of bio and chemical fertilizers on the growth and essential oil yield of fennel Foeniculum vulgare, Mill. Alex.J.Agric.Res., 49 (3):8391.

A. O. A. C. (1970). Official Methods of Analysis of Association of Official Agricultural
Chemists. Washington, D. C., $10^{\text {th }}$ ed.

Ayub M. A. A., Nadeem M., Tanveer M.T., Tahir y., Saqib and Nawaz R. (2008). Effect of different sowing methods and times on the growth and yield of fennel (Foeniculum vulgare Mill) Pak. J. Bot., 40 (1): 259-264.

Badawi A. M. (2000). Effect of some agriculture treatments on growth and active ingredients in roselle plants in North Sinai. Ph. D. Thesis, Fac. Agric., Zagazig Univ.,Egypt.

Bishr G. A. A., El-Mogy E.A.M. and Ibrahem H.M.A. (2006). Effect of some organic manures and micronutrients on the growth, seed yield and chemical constituents of borage (Borago officinalis L.) plants under sandy soil conditions. Zagazig J. Agric. Res., 33 (6): 1089-1112.

Boulos L. (1983). Medicinal Plants of North Africa, Ref. Public, Inc. Clair River, Algonac, Michigan 4800 /p. 109.

Bown D. (1995). "In Encyclopedia of Herbs \& their Uses." The Royal Horticultural Society, Londen $-424 \mathrm{pp}$.

British Pharmacopia (1963). Determination of Volatile Oil in Drugs. The Pharmaceutical Press, London.

Chapman H. D. and Pratt P. F. (1961). Methods of Soil, Plants and Water Analysis. University of California, Division of Agricultural Sciences.

Chevallier A. (1996). In "The Encyclopedia of Medicinal Plants". United States, DK Publishing Inc., 95 Madison Avenue, New York ,10016. 336, pp.

Costat Software program (1986). For the design and analysis of agronomic research experiments. Chort. Software, Costat 3 - 30, Berkeley, CA., U.S.A.C.F.

Cottenie A., Verloo M., Velghe M. and Camerlynck R. (1982). Chemical Analysis of Plant and Soil. Laboratory of Analytical and Agrochemistry.State Univ. Ghent, Belgium.

El-Ghadban E. A. E., El-Tobgy K. M. K. and Soliman S. G. I. (2008). Response of lavander plant (Lavendula multifida, L.) to compost and active dry yeast. J. Product \& Dev., 13 (1): 79-99.

Eshed A (2007). Egyptian Spices and Herb Export Development Association and Egyptian Society for the Producers, Manufacturers and Exporters of Medicinal and Aromatic Plants (ESMAP), EGYPT-Dr. FaroukElshobaki, drfarouk@elshobaki.comWEB:Esheda@Li 
nk.Net and http://www.esmap.org.eg/

Formacek V. and Kubeczka K. H. (1982). Essential Oils Analysis by Capillary Gas Chromatography and Carbon-13 NMR spectroscopy, John Wiley \& Sons, New York (ALNAP Database Ref. ID: 171).

Gad W. M. (2001). Physiological studies on Foeniculum vulgare Mill. and Anethum graveolens L. M.Sc. Thesis., Fac. Agric., Kafr El-Sheikh, Tanta Univ., Egypt.

Hamed E. S. (2004). Studies on planting of some medicinal plants in the desert. M.Sc. Thesis, Fac. Agric, Kafr El-Sheikh, Tanta University., Egypt.

Ibrahem T. Z. (2000). Effect of some cultural practices on growth and chemical composition of some medicinal plants under conditions of Northern Sinai. Ph.D. Thesis, Fac. Agric., Kafr El-Sheikh, Tanta University. Egypt.

Jacoub R. W. (1995). Effect of chemical fertilization on growth and oil yield of sweet basil ( Ocimum basilicum, L. ) plants. M. Sc. Thesis, Fac. Agric., Cairo Univ., Egypt.

Lawless J. (1992). The Encyclopedia of Essential Oils .Element Books, Limited. Long meed,Shaftesbury. Dorest. Great Britain.

Leung A. Y. (1980). In "Encyclopedia of Common Natural Ingredients Used in Food, Drugs, and Cosmetics". John Wiley \& Sons, New York 409 pp.

Mahfouz S. A. S. and Sharaf-Eldin M. A. (2007). Effect of mineral vs. biofertilizer on growth, yield and essential oil content of fennel. Int. Agrophysics, 21, 361-366.

Market News Service (MNS) Medicinal Plants \& Extracts (2006). No. 20 September 2006 International Trade Centre UNCTAD/WTO54-56 rue de Montbrillant, CH-1202 Geneva, Switzerland.

Murphy J. and Riley J. H. (1962). A modified single solution for the determination of phosphate in natural waters. Annal. Chem. Acta, 27: 31-36.
Musa Ozcan, Jean-Claude Chalchat (2005). Effect of different locations on the chemical composition of essential oils of laurel (Laurus nobilis L.) leaves growing wild in Turkey. Journal of Medicinal Food. September 1, 2005, 8(3): 408-411.

Naguib Y. N., Hussein M. S., El-Sherbeny S. E., Khalil M.Y. and Lazari D. (2007). Response of Ruta graveolens L. to sowing dates and foliar micronutrients. Journal of Applied Science Research,3 (11 ): 15341543.

Osman Y. A. H (2000). Possibility of production of coriander (Coriander sativum L.) under Sinai conditions. Ph. D. Thesis, Fac. Agric., Cairo University.

Rashed N. M. M (2002). Effect of fertilization on the growth and storability of some aromatic plants. M. Sc. Thesis, Fac. Agric., Kafr ElSheikh, Tanta Univ., Egypt.

Razan H. A. (2010). The effect of sowing dates and potassium fertilization on the yield and the quality of the produced essential oil of Anise Plant (Pimpinella anisum L). M.Sc. Thesis, Fac. Agric., Damascus Univ., Syria.

Robert Adams (1995). Identification of Essential Oils by Gas Chromatography - Mass Spectrometry. Allurd Pub., USA.

Sadtler R. (1986). The Sadtler standard gaschromatography retention index library vol 4. Sadtler Research Laboratories, Philadelphia, Pennsylvania.

Shalan M. N., Abd-Ellatif T. A., Soliman S.G.I. and El-Gaawwas E.O. (2001). Effect of some chemical and biofertilizer treatments on roselle plants Hibiscus sabdariffa, L. Egyptian Journal of Agricultural Research. 79: 2, 587-606.

Simon J. E. (1990). Essential Oils and Culinary Herbs, p. 472-483. In: J. Janick and J.E. Simon (eds.). Advances in new crops. Timber Press, Portland, OR.

Snedecor G.W. and Cochran W.G. (1968). Statistical Methods, Sixth edition. The Iowa State Univ. Press, Ames, Iowa, U.S.A. 


$$
\begin{aligned}
& \text { تأثير مواعيد الزراعه ومعاملات التسميد الحيوى والعضوى و الكيماوى } \\
& \text { على النمو والاتتاج لنبات الثمر الهندى تحت ظروف شمال سيناء }
\end{aligned}
$$

ايمان مختار أبو الغيط - أنور عثمان جمعه- أحمد سعيد يوسف - *|لهام محمد عطيه - *وفاء حامد عبد العليم عبد الله قسم البساتين - كلية الزر اعة- جامعة بنها -* قسم النباتات الطبيه و العطريه -مركز بحوث الصحر اء - القاهره- مصر

\section{ملخص}

تمت هذه الدر اسة في محطة بحوث الثيخ زويد التابعة لمركز بحوث الصحر اء المّاء - محافظة شمال سيناء في موسمين

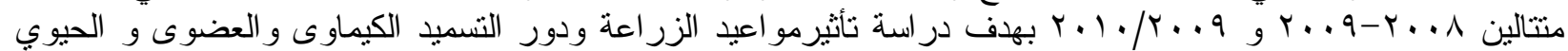

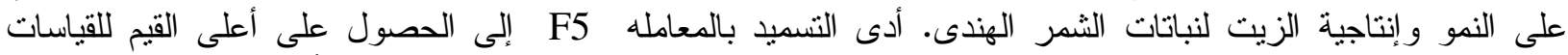

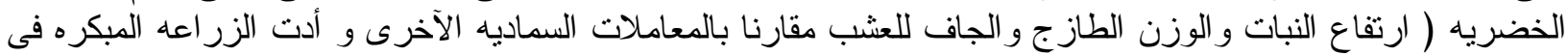

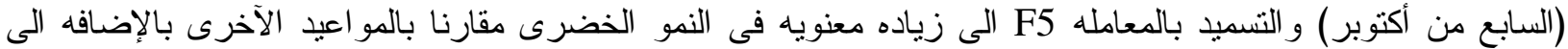

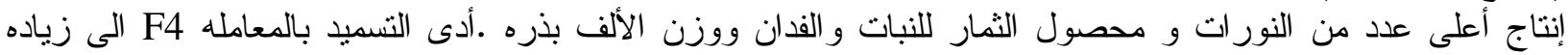

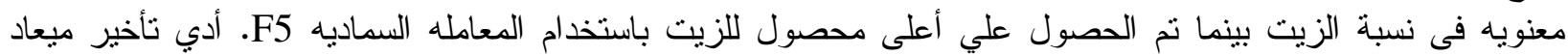

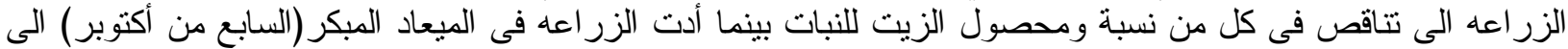

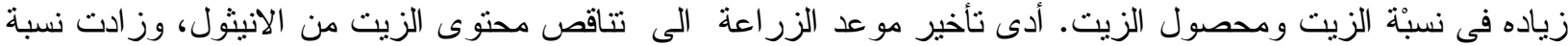

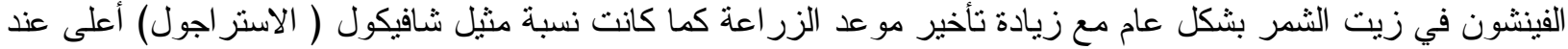

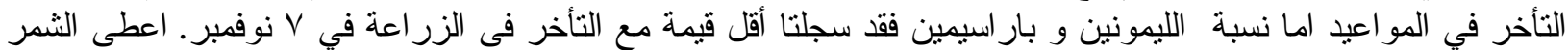

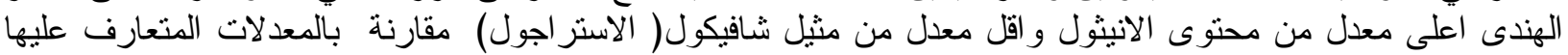

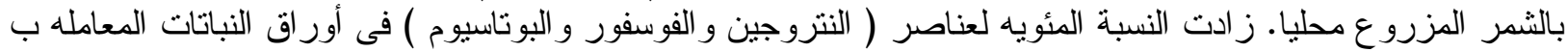
• ف5 ك5 كلا الموسمين 